\title{
Upper and lower spinal cord blood supply: The continuity of the anterior spinal artery and the relevance of the lumbar arteries
}

\author{
Paolo Biglioli, $\mathrm{MD}^{\mathrm{a}}$ \\ Maurizio Roberto, $\mathrm{MD}, \mathrm{PhD}^{\mathrm{a}}$ \\ Aldo Cannata, MD \\ Alessandro Parolari, MD, $\mathrm{PhD}^{\mathrm{a}}$ \\ Andrea Fumero, MD ${ }^{\mathrm{a}}$ \\ Francesco Grillo, MD \\ Marco Maggioni, MD \\ Guido Coggi, MD \\ Rita Spirito, $M^{a}$
}

From the Department of Cardiovascular Surgery, ${ }^{\mathrm{a}}$ University of Milan, Centro Cardiologico Fondazione Monzino IRCCS, Milan, Italy, and II Department of Pathology, ${ }^{\text {b }}$ University of Milan, San Paolo Hospital, Milan, Italy.

Received for publication Aug 31, 2003; revisions requested Nov 5, 2003; accepted for publication Nov 10, 2003.

Address for reprints: Aldo Cannata, MD, or Maurizio Roberto, MD, PhD, Department of Cardiovascular Surgery, Centro Cardiologico Fondazione Monzino IRCCS, Via Parea, 4, 20138 Milan, Italy (E-mail: aldo.cannata@libero.it; maurizio.roberto@ ccfm.it).

J Thorac Cardiovasc Surg 2004;127:1188-92 $0022-5223 / \$ 30.00$

Copyright $\odot 2004$ by The American Association for Thoracic Surgery

doi:10.1016/j.jtcvs.2003.11.038
Objective: Thoracic and thoracoabdominal aortic repair are still complicated by spinal cord ischemia and paraplegia. The aim of the present article is to present the results of an anatomical study conducted by means of both postmortem injection of the vertebral artery and perfusion of the abdominal aorta.

Methods: The spinal cord blood supply was investigated in 51 Caucasian cadavers: in 40 cases a methylene blue solution was hand-injected into the vertebral artery, whereas in the remaining 11 cases the abdominal aorta was perfused with a methylene blue solution by means of a roller pump. The level and side of the arteria radicularis magna and the continuity of the anterior spinal artery were recorded.

Results: The anterior spinal artery was a continuous vessel without interruptions along the spinal cord in all 51 cases. The arteria radicularis magna level was variable, ranging from $\mathrm{T} 9$ to $\mathrm{L} 5$. The arteria radicularis magna arose from a lumbar artery in 36 cases $(70.5 \%)$ and it was left-sided in 32 cases $(62.7 \%)$.

Conclusions: The anterior spinal artery constitutes an uninterrupted pathway between the vertebral arteries, the arteria radicularis magna, and the posterior intercostal and lumbar arteries. Moreover, the arteria radicularis magna arises from a lumbar artery in most of cases. Therefore, the sacrifice of the intercostal arteries during a thoracic aorta repair could be justified, at least from an anatomical standpoint. However, if an extended thoracoabdominal aortic repair is planned, it may be prudent to preserve the blood flow from the lumbar arteries.

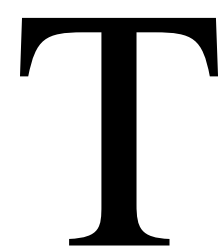

horacic and thoracoabdominal aortic repair for aneurysmal disease may be complicated by spinal cord ischemia and paraplegia. The anatomic bases and the pathophysiology of this severe complication are debated. Controversies still concern the anatomical arrangement of the anterior spinal artery (ASA) and the arteria radicularis magna (ARM) or Adamkiewicz artery. The aim of the present article is to describe the blood supply of the spinal cord from an anatomical point of view by means of postmortem injection of the vertebral arteries (upper blood supply) and the perfusion of the abdominal aorta (lower blood supply).

\section{Materials and Methods}

From July 1998 to July 2001, necropsy was performed on 51 Caucasian adult cadavers at II Department of Pathology of the University of Milan. Preliminary results that emerged from 


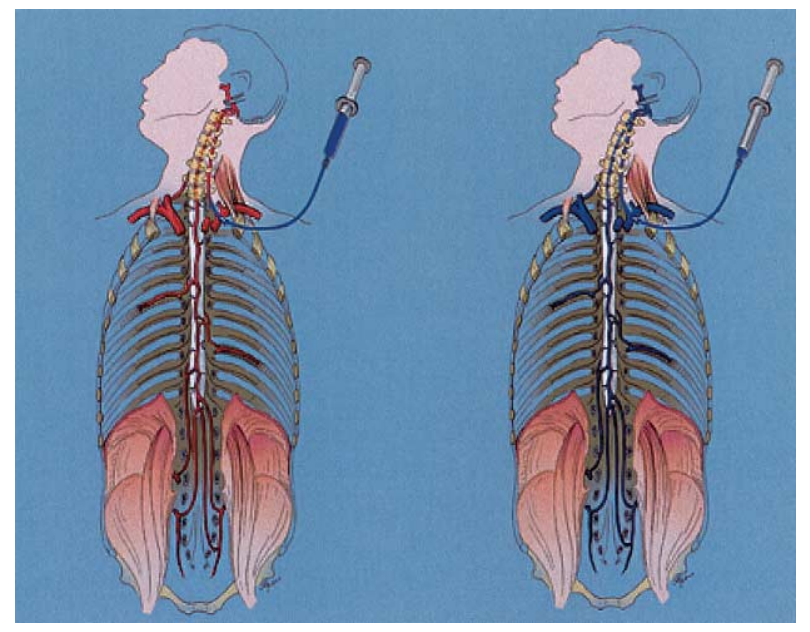

Figure 1. The dye injected through the left vertebral artery progressively stains the human spinal arteries.

the study of the first 31 cases were previously published. ${ }^{1}$ There were 35 men $(68.6 \%)$ and 16 women $(31.4 \%)$. The mean age was $72.9 \pm 12.4$ years. The necropsies were performed 24 to 36 hours after death. Gross neurological disease and aneurysmal or dissecting aortic disease were not observed in any case. At least mild atherosclerotic changes of the aorta and the cerebrovascular arteries were present in all cases.

\section{Upper Spinal Cord Blood Supply: Vertebral Artery Injection}

The technique of vertebral artery injection was previously described. ${ }^{1}$ Briefly, in 40 cases, after spinal cord exposure we injected into a vertebral artery (left-sided in 23 cases [57.5\%] and right-sided in 17 cases [42.5\%]) a solution containing polygelatincolloid and $10 \mathrm{~mL}$ of $1 \%$ methylene blue by gentle hand pressure. During the injection the staining of the spinal vessels was observed and recorded (Figure 1).

\section{Lower Spinal Cord Blood Supply: Perfusion of the Abdominal Aorta}

In 11 cases it was possible to remove the thoracic and abdominal viscera leaving intact in situ the entire descending thoracic and the abdominal aorta and their branches. Anatomical dissection was performed carefully to preserve the integrity of most of the collateral networks between intercostal, lumbar, and radiculomedullary arteries. First and second thoracic vertebral bodies were removed by means of an oscillating saw, and the dura mater was incised longitudinally to expose the upper thoracic ASA. Then all the posterior intercostal arteries were ligated and divided just at the origin from the aorta and before their first branching, as previously described in the quick simple clamping technique. ${ }^{2}$ The celiac trunk and mesenteric and renal arteries were surgically interrupted, and lumbar arteries were left intact. The common iliac arteries were divided just proximally to the origin of the hypogastric arteries. A $20 \mathrm{~F}$ or $22 \mathrm{~F}$ venous cannula, $40 \mathrm{~cm}$ in length (Terumo

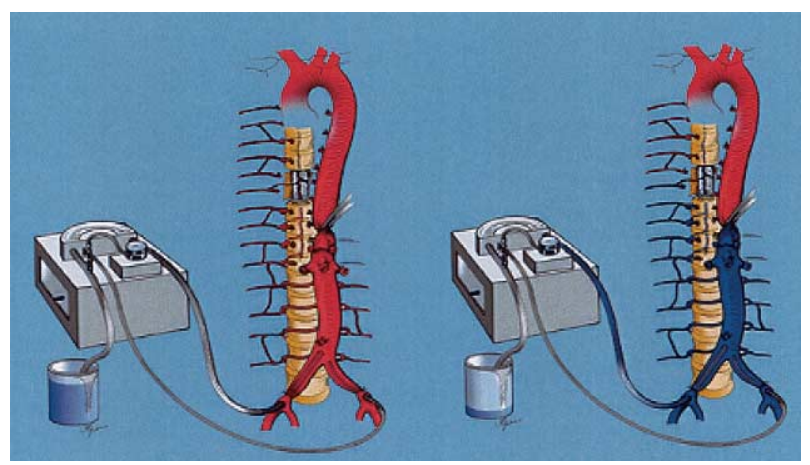

Figure 2. The dye injected through the common iliac artery progressively stains the abdominal aorta, the lumbar and intercostals arteries, and the anterior spinal artery.

Europe, Rome branch, Italy), was introduced into the proximal stump of the right or left common iliac artery, and the controlateral iliac artery was connected to a $16 \mathrm{~F}$ catheter, $25 \mathrm{~cm}$ in length, for coronary perfusion (Terumo Europe, Rome, Italy). By means of tubing (Tygon tube $3 / 8 \times 9 / 16 \times 3 / 32$ inches, $1 \mathrm{~m}$ in length; HST tube $1 / 4 \times 3 / 8 \times 1 / 16$ inches, $1 \mathrm{~m}$ length; NGC Medical Equipment, Novedrate, Italy), the inflow cannula was connected to a roller pump (Gambro, Parma, Italy) and to a reservoir with 1-L capacity, whereas the $16 \mathrm{~F}$ catheter was joined to a disposable pressure display set (Edwards Lifescience, Milan, Italy) to monitor the inflow pressure. First, air and clots were flushed from the tubing and the aorta with 500 to $750 \mathrm{~mL}$ of normal saline solution. During this phase, we checked any fluid loss and eventually closed leaks from the vessels to obtain a watertight system. Then, the aorta was clamped at the diaphragm and $480 \mathrm{~mL}$ of polygelatin colored with $20 \mathrm{~mL}$ of $1 \%$ methylene blue was injected and the mean inflow pressure was recorded over a 5-minute period (Figure 2). Simultaneously, the progression of the dye along the ASA was recorded using a video camera. After the procedure, 2 independent observers (M.R. and A.C.) reviewed each recording and measured the timing of ASA staining.

\section{Spinal Cord Inspection}

In all 51 cases the vertebral level of the highest thoracic root was recognized in situ and marked with a silk suture. Finally the spinal cord was removed from the cadaver. To establish the exact level of each anterior radicular artery, the filum terminalis and the spinal roots were arranged in the normal anatomical configuration and were accurately numerated starting from the filum, cross-checking the result with the level of the previously marked root. The largest anterior radicular artery supplying the majority of the lumbar enlargement, independently from the level, was recognized as the ARM. The level of the ARM and the other radicular artery was recorded, and in all cases the external diameters of the ASA and ARM were measured with a Palmer-type micrometer. The entire dissection was performed with the aid of $\times 3.5$ optical magnification. The diameters of ASA and ARM were compared by the Student $t$ test. 


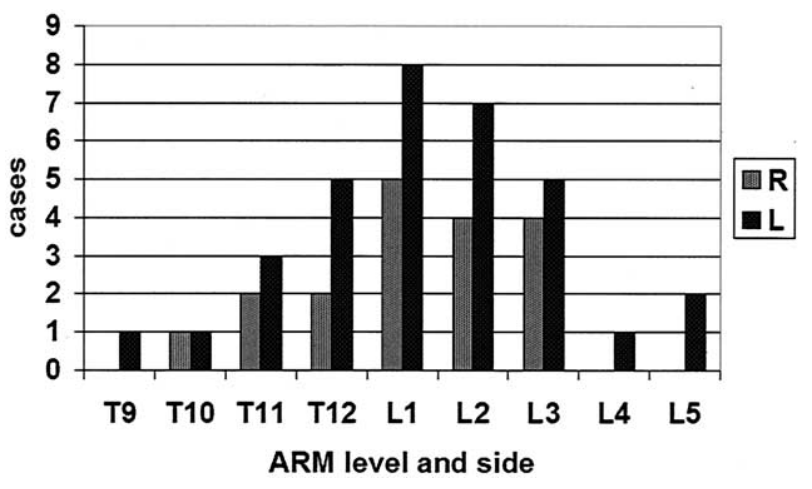

Figure 3. Level and side of the arteria radicularis magna (ARM) (n $=51$ ).

\section{Results \\ Upper Spinal Cord Blood Supply: Vertebral Artery Injection}

After hand injection of the methylene blue solution, we observed in every case the progressive staining first of both vertebral arteries, then of the ASA in cranio-caudal direction down to the cauda equina, and finally the anterior radicular arteries, including the ARM, stained retrogradely from the ASA. The side (left vs right) of the injected vertebral artery had no influence on the staining of the ASA.

\section{Lower Spinal Cord Blood Supply: Perfusion of the Abdominal Aorta}

At a mean inflow pressure of $65 \mathrm{~mm} \mathrm{Hg}$ the dye gradually stained the abdominal aorta and the lumbar arteries and finally the intercostal arteries even if previously interrupted and if aorta was clamped at the diaphragm. The lumbar arteries were directly stained from the abdominal aorta and indirectly from vertical arterial branches, although the intercostal vessels were supplied only by the arteries running lateral to the vertebral bodies and joining them perpendicularly. These vessels formed an impressive and extensive collateral network over the entire vertebral column. The colored solution gradually stained the radiculomedullary arteries and the ASA in a caudo-cranial direction. If the ARM originated from a lumbar artery, the ASA colored in about 1 to 2 minutes after start of perfusion, although if it arose from an intercostal artery, the ASA was stained only after 5 to 6 minutes.

\section{Spinal Cord Inspection}

At the necropsy examination, the ASA was a constant and continuous vessel without interruption along the spinal cord in all 51 cases. No case presented a duplication of the ASA. Only 1 ARM was constantly found in each cadaver and it was the only radicular artery to the mid and low thoracic and lumbar spinal cord in 16 cases (31.3\%). In all cases it was the more caudal anterior radicular artery of significant diameter. Figure 3 shows the vertebral level and the lateralization of the ARM in the series. In 36 cases (70.5\%) the ARM arose below T12 level, from a lumbar artery, and its origin was more frequently located at L1-L3 level (33 cases, $64.7 \%)$. The Adamkiewicz artery showed a left-side predominance, observed in 32 cadavers $(62.7 \%)$. The mean external diameter of the ASA was $480 \pm 81 \mu \mathrm{m}$ (range: 340-560 $\mu \mathrm{m}$ ) and $1100 \pm 132 \mu \mathrm{m}$ (range: 870-1280 $\mu \mathrm{m}$ ) above and below with the junction with the ARM, respectively $(P<.001)$. The mean external diameter of the ARM was $1120 \pm 230 \mu \mathrm{m}$ (range: $730-1330 \mu \mathrm{m}$ ).

\section{Discussion}

There is still disagreement about the anatomy of the spinal cord arterial supply. Main issues concern the existence and the terminality of the ARM, or artery of Adamkiewicz, and the continuity of the ASA. ${ }^{1-10}$ Techniques adopted in clinical practice are founded both on evidence from postmortem anatomical investigations conducted on human beings ${ }^{11,12}$ and from experimental studies conducted on animals. ${ }^{13-15}$ Nevertheless, comparative anatomy suggests major differences between human beings and animals in terms of spinal cord blood supply ${ }^{16}$ and, to the best of our knowledge, only the results from baboons are extremely similar to humans. ${ }^{11}$ Consequently, evidence from most of the studies on animals may not reflect the results observed in clinical practice.

We focused our observations on the mid and low thoracic and lumbar spinal cord because postoperative ischemic lesions are located in this site. ${ }^{17}$ Our anatomical observations confirm that the ASA is constantly an uninterrupted vessel receiving blood supply from the vertebral arteries, the ARM, and the other anterior radiculomedullary arteries. All these vessels are interconnected to form an extensive collateral network over the entire vertebral column, easily recognizable during dye injection. Because of the continuity of the ASA, the ARM cannot be considered a terminal artery per se. This observation is in agreement with the experimental studies on rhesus monkeys by Fried and colleagues. ${ }^{18}$

In our series the ARM level ranged from T9 to L5 and was located below T12 in 70.5\% of cases and between L1 and L3 in $64.7 \%$ of cases. These results are in agreement with the observation reported by Suh and Alexander ${ }^{8}$ and Gillilan. ${ }^{19}$ Other authors reported that the more frequent level of the ARM was between T12 and L1., ,8,9,11,19 This disagreement may depend on the method applied to establish the ARM level. In our opinion, in comparison to a generic "vertebral level," an accurate reference to the spinal roots allows ascertainment of the exact level of the intercostal or lumbar artery from which ARM arises. In contrast to Koshino and colleagues. ${ }^{12}$ we observed an Adamkiewicz artery below L2 in $23.5 \%$ of cases (12/51), in absence of significant (more than $500 \mu \mathrm{m}$ in diameter) anterior radic- 
ular arteries located more cranially. Nevertheless, we cannot rule out differences in terms of spinal cord blood supply between Japanese and Caucasian populations.

\section{Implications for Surgical and Endovascular Therapy}

The continuity of the ASA, the nonterminality of the ARM, and the extensive collateral network along the spinal cord could justify from the anatomical standpoint the systematic sacrifice of posterior intercostal arteries during isolated repair of the descending thoracic aorta. However, because of the prevalent origin of the ARM from lumbar arteries in addition to previously reported clinical experiences, ${ }^{20}$ it may be prudent to preserve blood flow from the lumbar arteries when an extensive thoracoabdominal aortic repair is planned. In fact, there is increasing concern that concomitant or previous abdominal aortic repair and extensive thoracic aorta exclusion by means of multiple stent grafts are associated with an significantly higher risk of paraplegia. ${ }^{21-23} \mathrm{We}$ can hypothesize that after the interruption of the most of the intercostal and lumbar arteries, the residual collateral blood supply is marginal and in some cases the spinal cord may become extremely prone to injury due to arterial hypotension or low cardiac output from any cause. $^{24,25}$ It is still debated whether the reimplantation of segmental vessels, usually focused on the intercostal vessels, may reduce the incidence of paraplegia. Routine reimplantation of the segmental arteries from $\mathrm{T} 7$ to $\mathrm{L}^{26}$ may miss the origin branch of ARM in at least $45 \%$ of cases, according to our results. Nevertheless, intercostal reimplantation may be of critical importance in patients undergoing thoracic repair after a previous abdominal aortic procedure.

Moreover, we have to recognize the other side of the coin about the continuity between the ASA and the radicular arteries. Both experimental ${ }^{15}$ and clinical ${ }^{27,28}$ data suggest that the blood may drain away from the spinal cord through the ASA and the radicular arteries, acting as stealing channels and rerouting the blood just distal to the aortic obstruction (Figure 4). Back-bleeding from the ostia of the posterior intercostal and lumbar arteries during aortic crossclamping may be a clinical manifestation of such a phenomenon. We can speculate that such continuous steal through the ASA and radicular vessels may further worsen spinal cord ischemia, causing even irreversible neurological injury if the ischemia time is longer than 20 to $30 \mathrm{~min}$ utes. ${ }^{29,30}$ Prolonged crossclamping time is a recognized risk factor for paraplegia in most of reports, ${ }^{31-34}$ and in our opinion it may be the most important contributor to postoperative paraplegia.

Finally, in a third of cases the ARM arose from the thoracic aorta; this anatomical arrangement may reduce the clinical efficacy of a circulatory support during aortic crossclamping. In fact, during postmortem perfusion of the abdominal aorta, we observed that the thoracic ASA was

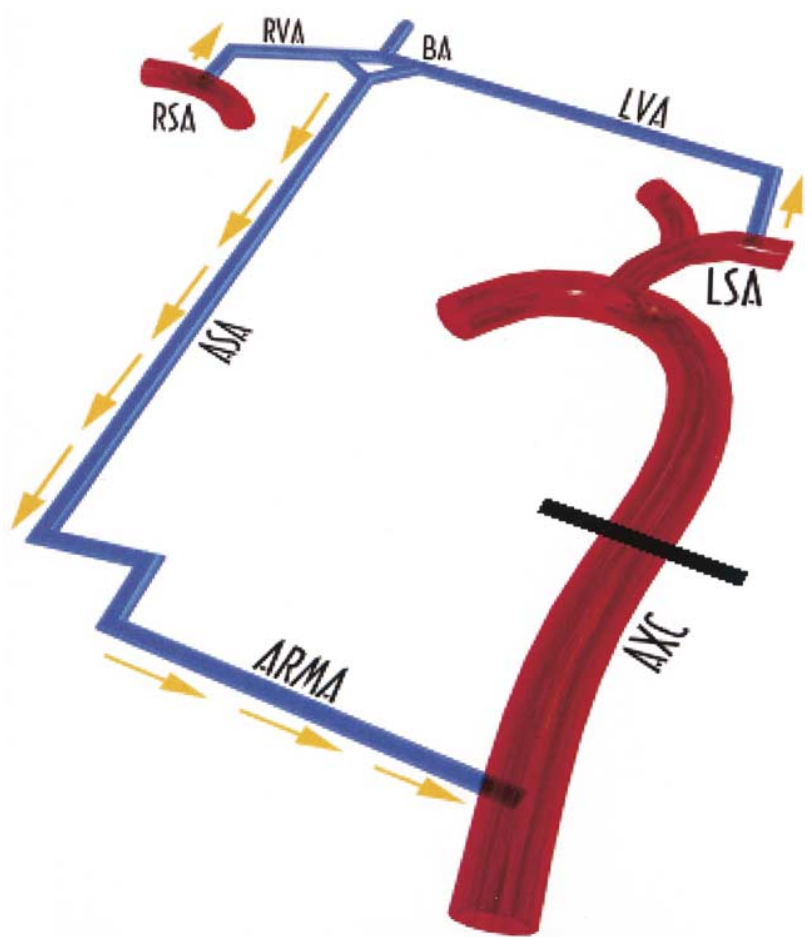

Figure 4. Steal phenomenon in the excluded part of the aorta. A rerouting way through the anterior spinal artery and arteria radicularis magna steals blood from the spinal cord. ASA, Anterior spinal artery; $A R M A$, anterior radicularis magna artery; $L S A$, left subclavian artery; $R S A$, right subclavian artery; $L V A$, left vertebral artery; $R V A$, right vertebral artery; $B A$, basilar artery; $A X C$, aortic clamping.

stained within 1 to 2 minutes after start of perfusion if the ARM originated from a lumbar artery; however, such time was raised to 5 to 6 minutes if the ARM arose from an intercostal artery.

\section{Limitations of the Study}

Abdominal aorta perfusion was feasible in only 11 cases. The most frequent reasons making abdominal aorta perfusion unfeasible were severe calcification of the iliac arteries and the aorta, impossibility of achieving a watertight system during perfusion because of irreparable leaks from several vessels, and finally the need to remove the aorta en bloc with the thoracoabdominal viscera on request of the pathologist to identify the cause of death and/or to perform special pathological investigations.

The injection and perfusion techniques were not conceived to simulate normal hemodynamics. Finally, in our series of necropsies we observed no case of aortic aneurysm, either thoracic or abdominal. It may be very useful to study the blood supply and the collateral circulation of the spinal cord in the presence of degenerative atherosclerotic or dissecting aneurysm or after a surgical or endovascular 
aortic procedure. In such patients the disease and the surgical procedure may occlude many segmental arteries and promote collateral vessels enlargement, altering significantly the normal patterns of blood supply to the spinal cord.

\section{Conclusions}

Anatomical evidence of an extensive collateral network along the spinal cord suggests that during a repair of the descending thoracic aorta the systematic sacrifice of segmental arteries may be considered safe. Nevertheless, we hypothesize that during aortic crossclamping this network may act itself as stealing pathway, rerouting the blood away from spinal cord to the aorta distal to the clamps. The exclusion of most of the segmental arteries during repair of thoracoabdominal aortic aneurysms may compromise the collateral blood supply of the spinal cord. In these cases every effort should be made to preserve the blood flow from the lumbar arteries.

We acknowledge Mrs Elena Greco and Miss Laura Barili for their professional artwork, and the staff of the Perfusion Service of Centro Cardiologico Monzino, Milan, and the Bioengineering Department of Politecnico of Milan for their invaluable help in providing materials, assistance, and suggestions for abdominal aortic perfusion.

\section{References}

1. Biglioli P, Spirito R, Roberto M, Grillo F, Cannata A, Parolari A, et al. The anterior spinal artery: the main arterial supply of the human spinal cord-a preliminary anatomic study. J Thorac Cardiovasc Surg. 2000; 119:376-9.

2. Biglioli P, Spirito R, Porqueddu M, Agrifoglio M, Pompilio G, Parolari A, et al. Quick, simple clamping technique in descending thoracic aortic aneurysm repair. Ann Thorac Surg. 1999;67:1038-43.

3. Adams HD. Neurologic complications of aortic surgery. Ann Surg. 1956;144:574-610.

4. Lazorthes G, Gouaze A, Zadeh O. Arterial vascularisation of the spinal cord. Recent studies of the anastomotic pathways. J Neurosurg. 1971; 35:253-62.

5. Brewer LA, Fosburg RG, Mulder GA, Verska JJ. Spinal cord complications following surgery for coarctation of the aorta. A study of 66 cases. J Thorac Cardiovasc Surg. 1972;64:368-81.

6. Thron AK. Vascular anatomy of the spinal cord. New York: Springer Verlag; 1988.

7. Berenstein A, Lasjaunias P. Surgical neuroangiography. Wein: Springer-Verlag; 1990.

8. Suh TH, Alexander L. Vascular system of the human spinal cord. Arch Neurol Psych. 1939;41:659-77.

9. Dommisse GF. The blood supply of the spinal cord. A critical vascular zone in spinal surgery. J Bone Joint Surg Br. 1974;56:225-35.

10. Tveten L. Spinal cord vascularity. III. The spinal cord arteries in man. Acta Radiol Diagn (Stockh). 1976;17:257-73.

11. Svensson LG, Klepp P, Hinder RA. Spinal cord anatomy of the baboon-comparison with man and implications for spinal cord blood flow during thoracic aortic cross-clamping. S Afr J Surg. 1986;24:32-4.

12. Koshino T, Murakami G, Morishita K, Mawatari T, Abe T. Does the Adamkiewicz artery originate from the larger segmental arteries? J Thorac Cardiovasc Surg. 1999;117:898-905.

13. Wadou F, Arndt CF, Oppermann E, Borst HG, Wadou R. The mech- anism of spinal cord injury after simple and double aortic crossclamping. J Thorac Cardiovasc Surg. 1986;92:121-7.

14. Harakawa I, Yano T, Sakurai T, Nishikimi N, Nimura Y. Measurement of spinal cord blood flow by an inhalation method and intraarterial injection of hydrogen gas. J Vasc Surg. 1997;26:623-8.

15. Christiansson L, Ulus AT, Hellberg A, Bergqvist D, Wiklund L, Karacagil S. Aspects of the spinal cord circulation as assessed by intrathecal oxygen tension monitoring during various arterial interruptions in the pig. J Thorac Cardiovasc Surg. 2001;121:762-72.

16. Lazorthes G, Gouazè A, Djindjian R. Vascularisation et circulation de la moelle épinière. Paris: Masson; 1973.

17. Mawad ME, Rivera V, Crawford S, Ramirez A, Breitbach W. Spinal cord ischemia after resection of thoracoabdominal aortic aneurysms: MR findings in 24 patients. AJR Am J Roentgenol. 1990;155:1303-7.

18. Fried LC, Di Chiro G, Doppman JL. Ligation of major thoraco-lumbar spinal cord arteries in monkeys. J Neurosurg. 1969;31:608-14.

19. Gillilan LA. The arterial blood supply of the human spinal cord. J Comp Neurol. 1958;110:75-103.

20. Griepp RB, Ergin MA, Galla JD, Lansman S, Khan N, Quintana C, et al. Looking for the artery of Adamkiewicz: a quest to minimize paraplegia after operations for aneurysms of the descending thoracic and thoracoabdominal aorta. J Thorac Cardiovasc Surg. 1996;112: 1202-13.

21. Buffolo E, da Fonseca JH, de Souza JA, Alves CM. Revolutionary treatment of aneurysms and dissections of descending aorta: the endovascular approach. Ann Thorac Surg. 2002;74:S1815-7.

22. Mitchell RS, Miller DC, Dake MD, Semba CP, Moore KA, Sakai T. Thoracic aortic aneurysm repair with an endovascular stent graft: the "first generation." Ann Thorac Surg. 1999;67:1971-4.

23. Gravereaux EC, Faries PL, Burks JA, Latessa V, Spielvogel D, Hollier $\mathrm{LH}$, et al. Risk of spinal cord ischemia after endograft repair of thoracic aortic aneurysms. J Vasc Surg. 2001;34:997-1003.

24. Ishimaru S, Kawaguchi S, Koizumi N, Obitsu Y, Ishikawa M. Preliminary report on prediction of spinal cord ischemia in endovascular stent graft repair of thoracic aortic aneurysm by retrievable stent graft. J Thorac Cardiovasc Surg. 1998;115:811-8.

25. Acher CW, Wynn MM, Hoch JR, Kranner PW. Cardiac function is a risk factor for paralysis in thoracoabdominal aortic replacement. $J$ Vasc Surg. 1998;27:821-8.

26. Svensson LG. Commentary to: Koshino T, Murakami G, Morishita K, Mawatari T, Abe T. Does the Adamkiewicz artery originate from the larger segmental arteries? J Thorac Cardiovasc Surg. 1999;117:903-5.

27. Darwish H, Archer C, Modin J. The anterior spinal artery collateral in coarctation of the aorta. A clinical angiographic correlation. Arch Neurol. 1979;36:240-3.

28. Kendall BE, Andrew J. Neurogenic intermittent claudication associated with aortic steal from the anterior spinal artery complicating coarctation of the aorta. J Neurosurg. 1972;37:89-94.

29. Van Harreveld A, Schadé JP. Nerve cell destruction by asphyxiation of the spinal cord. J Neuropathol Exp Neurol. 1962;21:410-22.

30. Taira Y, Marsala M. Effect of proximal arterial perfusion on function, spinal cord blood flow, and histopathologic changes after increasing intervals of aortic occlusion in the rat. Stroke. 1996;27:1850-8.

31. Livesay JJ, Cooley DA, Ventemiglia RA, Montero CG, Warrian RK, Brown DM, et al. Surgical experience in descending thoracic aneurysmectomy with and without adjuncts to avoid ischemia. Ann Thorac Surg. 1985;39:37-46.

32. Katz NM, Blackstone EH, Kirklin JW, Karp RB. Incremental risk factors for spinal cord injury following operation for acute traumatic aortic transection. J Thorac Cardiovasc Surg. 1981;81:669-74.

33. Svensson LG, Hess KR, Coselli JS, Safi HJ. Influence of segmental arteries, extent, and atriofemoral bypass on postoperative paraplegia after thoracoabdominal aortic operations. J Vasc Surg. 1994;20:25562.

34. Cambria RP, Davison JK, Zannetti S, L'Italien G, Atamian S. Thoracoabdominal aneurysm repair: perspectives over a decade with the clamp-and-sew technique. Ann Surg. 1997;226:294-303. 